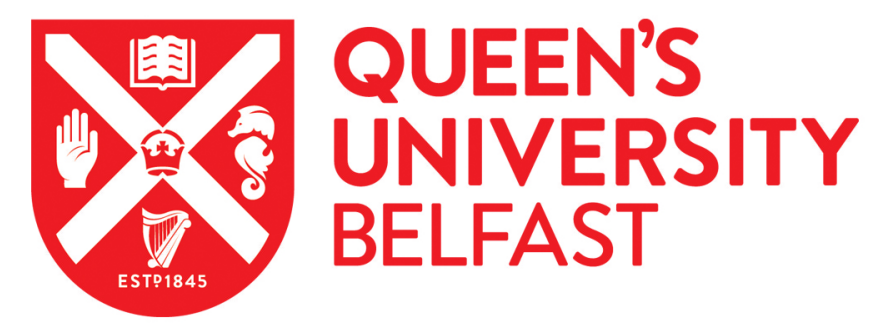

\title{
Observation and characterization of laser-driven Phase Space Electron Holes
}

Sarri, G., Dieckmann, M. E., Brown, C. R. D., Cecchetti, C., Hoarty, D. J., James, S. F., Jung, R., Kourakis, I., Schamel, H., Willi, O., \& Borghesi, M. (2010). Observation and characterization of laser-driven Phase Space Electron Holes. Physics of Plasmas, 17(1), [010701]. https://doi.org/10.1063/1.3286438

Published in:

Physics of Plasmas

Document Version:

Publisher's PDF, also known as Version of record

Queen's University Belfast - Research Portal:

Link to publication record in Queen's University Belfast Research Portal

Publisher rights

(C) 2010 American Institute of Physics

\section{General rights}

Copyright for the publications made accessible via the Queen's University Belfast Research Portal is retained by the author(s) and / or other copyright owners and it is a condition of accessing these publications that users recognise and abide by the legal requirements associated with these rights.

Take down policy

The Research Portal is Queen's institutional repository that provides access to Queen's research output. Every effort has been made to ensure that content in the Research Portal does not infringe any person's rights, or applicable UK laws. If you discover content in the Research Portal that you believe breaches copyright or violates any law, please contact openaccess@qub.ac.uk. 


\title{
Observation and characterization of laser-driven phase space electron holes
}

\author{
G. Sarri, ${ }^{1}$ M. E. Dieckmann, ${ }^{2}$ C. R. D. Brown, ${ }^{3}$ C. A. Cecchetti, ${ }^{1}$ D. J. Hoarty, ${ }^{3}$ \\ S. F. James, ${ }^{3}$ R. Jung, ${ }^{4}$ I. Kourakis, ${ }^{1}$ H. Schamel,${ }^{5}$ O. Willi, ${ }^{4}$ and M. Borghesi ${ }^{1}$ \\ ${ }^{1}$ School of Mathematics and Physics, The Queen's University of Belfast, Belfast BT7 1NN, United Kingdom \\ ${ }^{2}$ ITN, Linkoping University, 60174 Norrkoping, Sweden \\ ${ }^{3}$ AWE, Aldermaston, Reading, Berkshire RG7 4PR, United Kingdom \\ ${ }^{4}$ Institute for Laser and Plasma Physics, Heinrich-Heine-University, 40225 Düsseldorf, Germany \\ ${ }^{5}$ Physikalisches Institut, Universität Bayreuth, D-95440 Bayreuth, Germany
}

(Received 12 November 2009; accepted 15 December 2009; published online 7 January 2010)

\begin{abstract}
The direct observation and full characterization of a phase space electron hole (EH) generated during laser-matter interaction is presented. This structure, propagating in a tenuous, nonmagnetized plasma, has been detected via proton radiography during the irradiation with a ns laser pulse $\left(I \lambda^{2} \approx 10^{14} \mathrm{~W} / \mathrm{cm}^{2}\right)$ of a gold hohlraum. This technique has allowed the simultaneous detection of propagation velocity, potential, and electron density spatial profile across the EH with fine spatial and temporal resolution allowing a detailed comparison with theoretical and numerical models.
\end{abstract}

(C) 2010 American Institute of Physics. [doi:10.1063/1.3286438]

Phase-space electron holes ${ }^{1}$ (EHs) are electrostatic excitations in collisionless plasmas characterized by a positive potential hump in which a population of electrons is trapped. In addition to their relevance to many fundamental plasma processes such as two-stream instabilities ${ }^{2}$ and saturation in Landau damping, ${ }^{3}$ EH play a key role in a wide range of space plasma scenarios (e.g., microscopic dissipation during magnetic reconnection in the Earth's magnetosphere ${ }^{4}$ or the generation of cosmic ray electrons in supernovae ${ }^{5}$ ) and are commonly detected in near Earth plasmas ${ }^{6-8}$ in particular: auroral zone,${ }^{9}$ magnetosheath, ${ }^{10}$ magnetopause, ${ }^{11}$ bow shock transition region, ${ }^{12}$ and solar wind. ${ }^{13}$ The detrimental effect they have on the focusing properties of particle accelerators and storage rings has also been recently highlighted. ${ }^{14}$ The omnipresence of such structures in collisionless plasmas warrants therefore, beside in-depth theoretical modeling, ${ }^{1}$ equally detailed experimental investigations. Previous experimental work detected this type of structures in magnetized collisionless plasmas either in Q-machines ${ }^{15,16}$ or during magnetic reconnection in toroidal plasma current sheets. ${ }^{17}$ In all these cases the existence of EH was deduced from positive spikes in high-bandwidth Langmuir probes. Advancing these previous detections, here we report the first direct observation of an EH in a laser-matter interaction experiment, and suggest a new way to generate and study them in a controllable manner. EH diagnosis using proton projection imaging (PPI) technique ${ }^{18,19}$ allows in fact the simultaneous measurement of propagation velocity, potential, and electron density spatial profile together with its temporal evolution leading to a detailed characterization with high resolution; this has allowed a detailed comparison with recently published theoretical modeling for EH behavior in nonMaxwellian plasmas.

The experiment, performed at the HELEN laser system in AWE ${ }^{20}$ involved the illumination of the inner surface of a hohlraum target by an intense and relatively long $(\tau \approx 1 \mathrm{~ns}$ temporally flat-top, $I \approx 10^{14} \mathrm{~W} / \mathrm{cm}^{2}, \lambda=0.527 \mathrm{~nm}$ ) laser pulse. The hohlraum target consisted of an open-ended $\mathrm{Au}$ cylinder with diameter of $1.5 \mathrm{~mm}$, length of $1 \mathrm{~mm}$, and wall thickness of $26 \mu \mathrm{m}$ (Fig. 1). The interaction beam [EAST beam in Fig. 1(a)] was focused through the laser entrance hole (LEH) onto the inner surface of the hohlraum. A second short and intense pulse $\left[\tau \approx 700 \mathrm{fs}, I>10^{19} \mathrm{~W} / \mathrm{cm}^{2}\right.$, CPA beam in Fig. 1(a)] was focused onto a $20 \mu \mathrm{m}$ gold foil in order to create, via target normal sheath acceleration, ${ }^{21}$ a wide spectrum proton beam. The proton beam, after having probed the plasma, was recorded on a stack of RadioChromic Films (RCF). ${ }^{22}$

Such a probing scheme enables monitoring of the transverse electric field distribution inside the plasma by measuring the deflection of a proton beam as it passes through it. The high degree of laminarity of the beam ${ }^{23}$ allows pointprojection imaging of the probed region.

Under the assumption of small deviations (i.e., the proton trajectories do not cross), the transverse electric field distribution can be derived directly from the relative modulation of the proton density deposited on a given RCF layer, ${ }^{18}$

$$
\left\langle E_{y}\right\rangle \approx-\frac{2 \varepsilon_{p} M}{e L b} \int \frac{\delta n_{p}}{n_{p}} d y,
$$

where $\langle E y\rangle$ is the transverse electric field component averaged along the longitudinal dimension, $\varepsilon_{p}$ is the probe proton energy, $L$ is the distance between the interaction area and the detector, $b$ is the longitudinal length of the nonzero electric field region, and $d n_{p} / n_{p}$ is the relative modulation of the proton density at the detector plane. Thanks to the broad spectrum of such a proton beam, combined to time-of-flight dispersion effects and energy resolution of the detector, different layers within the RCF stack provide snapshots of the interaction at different times even in a single shot configuration. ${ }^{19}$

Data exemplifying the features observed by PPI are dis- 


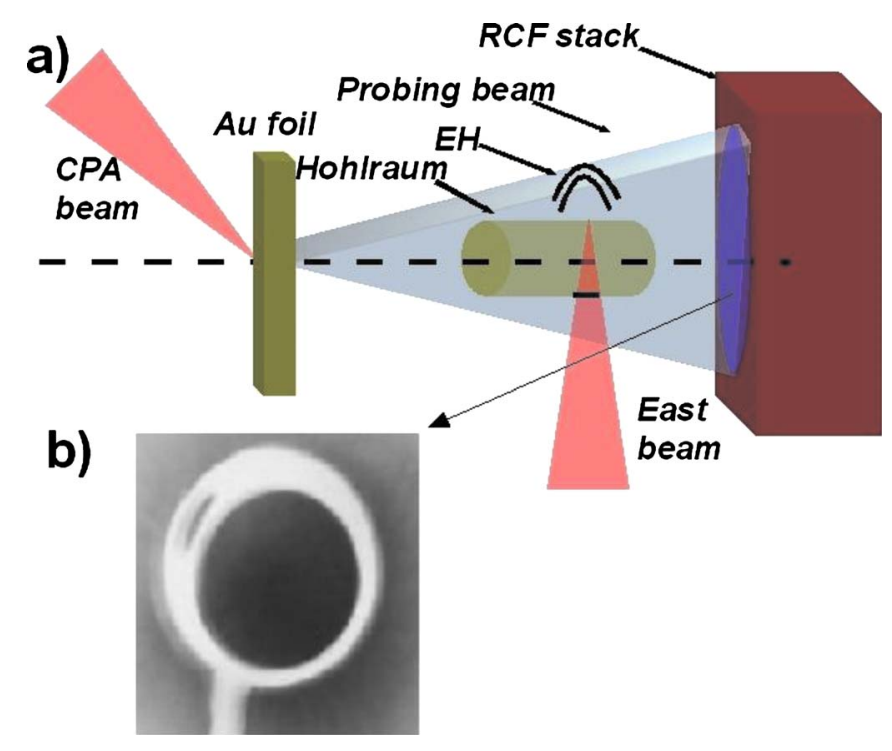

FIG. 1. (Color online) (a) Experimental setup: The EAST beam $\left(I \approx 10^{14} \mathrm{~W} / \mathrm{cm}^{2}, \tau \approx 1 \mathrm{~ns}\right.$ flat-top, $\left.\lambda \approx 0.527 \mu \mathrm{m}\right)$ irradiated the inner surface of a $1.5 \mathrm{~mm}$ diameter, $26 \mu \mathrm{m}$-thick open-ended gold hohlraum. The interaction was probed by the proton beam generated in the interaction between a $20 \mu \mathrm{m}$ gold foil and the CPA beam $\left(I \geq 10^{19} \mathrm{~W} / \mathrm{cm}^{2}\right.$, $\tau \approx 700 \mathrm{fs}$ ). (b) Proton radiograph of unirradiated hohlraum: the LEH is visible at the upper-left side of the hohlraum.

played in Figs. 2(a) and 2(b). As a general rule, the electric fields are directed from the regions of a lighter gray color compared to the background (reduced probe proton flux) toward the regions of darker gray color (increased flux). The feature that we discuss in the Letter, i.e., a region of pronounced modulation in the probe proton density [Fig. 2(c)], evidence of a modulated electric field distribution, is observed $\simeq 300-400 \mu \mathrm{m}$ from the rear surface of the irradiated target surface, well separated from the turbulent plasma observed at the interaction point.

By analysis of the different RCF layers within the same stack, this density modulation [shown in Fig. 2(c)] is seen to propagate with a constant velocity of $v \simeq(1.6 \pm 0.6)$ $\times 10^{6} \mathrm{~m} / \mathrm{s}$ (Fig. 3) while maintaining a substantially timeindependent profile in the comoving reference frame.

The electric field distribution across the structure, $E(x)$, has been extracted [Fig. 4(a)] using Eq. (1). The correspond- ing potential profile has been calculated by spatial integration of $E(x)$ [Fig. 4(b)]. In this calculation we have assumed a quasiplanar structure with longitudinal dimension $b$ of the order of the transverse dimension, i.e., $\approx 600 \mu \mathrm{m}$; these symmetry considerations concur with published numerical results. ${ }^{24}$ The potential exhibits a localized bell-shaped structure 80-90 $\mu \mathrm{m}$ wide with a maximum value of $\approx 30 \mathrm{~V}$.

In order to estimate the plasma parameters in the region of observation, the interaction between the laser and a $26 \mu \mathrm{m}$ thick gold foil has been simulated using a onedimensional (1D) hydrodynamic Lagrangian code (HYADES) including radiation transport and ionization. ${ }^{25}$ Simulations indicate that the energetic $\mathrm{x}$ rays generated during the interaction propagate through the gold foil and ionize the ambient gas at the rear surface (pressure $\simeq 10^{-3}$ mbar) creating a steady tenuous plasma; the electron temperature and density are predicted to be $n_{e} \simeq 2.5 \times 10^{12} \mathrm{~cm}^{-3}$ and $T_{e} \simeq 2 \mathrm{eV}$, respectively, implying a Debye length of $\lambda_{D} \simeq 7 \mu \mathrm{m}$, an electron plasma frequency of $\omega_{p e} \simeq 10^{11} \mathrm{~s}^{-1}$ (ion plasma frequency $\left.\omega_{p i} \simeq 5 \times 10^{8} \mathrm{~s}^{-1}\right)$, an electron thermal velocity of $v_{t h} \simeq 10^{6} \mathrm{~m} / \mathrm{s}$, and an ion acoustic velocity of $c_{s} \simeq 3$ $\times 10^{3} \mathrm{~m} / \mathrm{s}$. The velocity and the width of the structure are then $\simeq 1.6 v_{\text {th }}$ (or $\left.\simeq 530 c_{s}\right)$ and $\simeq 10-12 \lambda_{D}$, respectively, while the normalized maximum value of the potential is $\phi$ $=\mathrm{eV} / K_{B} T_{e} \simeq 15 \gg 1$ (thus lying above the limit for "weak" excitations ${ }^{1}$ ).

According to these parameters, the ratio of the electron mean free path to the electron Debye length is approximately $3 \times 10^{3}$. The plasma is then collisionless and it can thus support propagating EHs. ${ }^{1,24}$

Since the plasma is probed at $\approx 100-200$ ps after the beginning of interaction and the ion plasma frequency is $\omega_{p i} \simeq 5 \times 10^{8} \mathrm{~s}^{-1}\left(\omega_{p i}^{-1} \approx 2 \mathrm{~ns}\right)$, it is reasonable to neglect motion of the ions and to consider them as a still, neutralizing background in the plasma. Under this assumption, the electron density across the structure can be extracted from the data; the charge density, obtainable by Poisson's equation, is equal to $\rho(x)=e \cdot\left[n_{i}(x)-n_{e}(x)\right]$ where $n_{e}(x)\left[n_{i}(x)\right]$ is the electron (ion) density distribution. Simulations indicate an ionization state $Z=1$ therefore the ion density within the structure can be expressed as $n_{i}(x)=n_{i 0}=n_{e 0}$ leading to $n_{e}(x)=n_{i 0}-\rho(x) / e$, [Fig. 4(c)]. The electron density exhibits
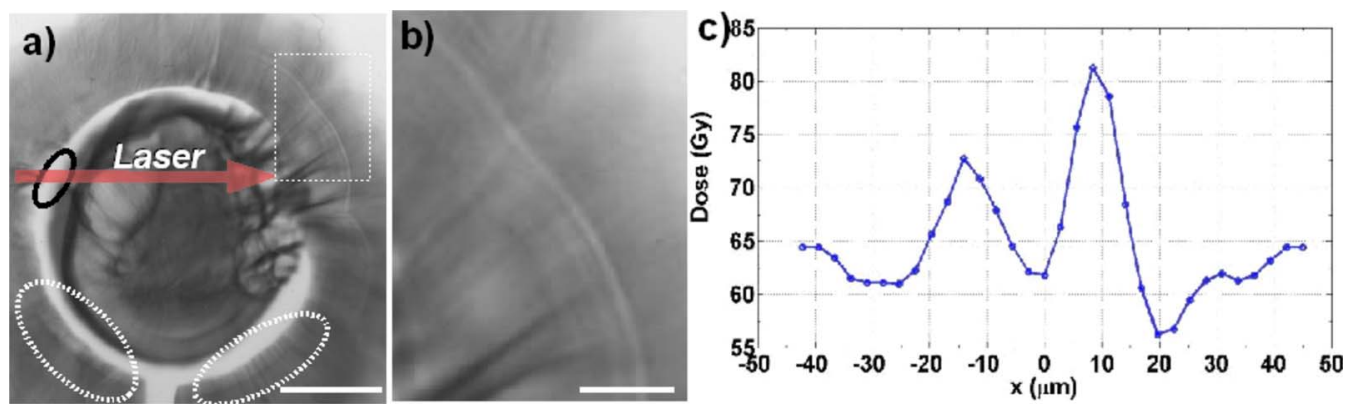

FIG. 2. (Color online) (a) Experimental data: RCF image associated to a time $\simeq 160 \mathrm{ps}$ after the beginning of interaction. The hohlraum is open-ended and has a diameter of $1500 \mu \mathrm{m}$. The dashed square in the top-right corner highlights the feature discussed in this letter. The white ellipses outline zones of proton density depletion due to the electrostatic charging of the hohlraum walls. The white ruler represents $500 \mu \mathrm{m}$. (b) Zoom of the structure. Zoom of the region outlined by the dashed square in (a).The white ruler represents $100 \mu \mathrm{m}$. (c) Proton signal: modulation in proton dose on the RCF layer in correspondence of the structure. 


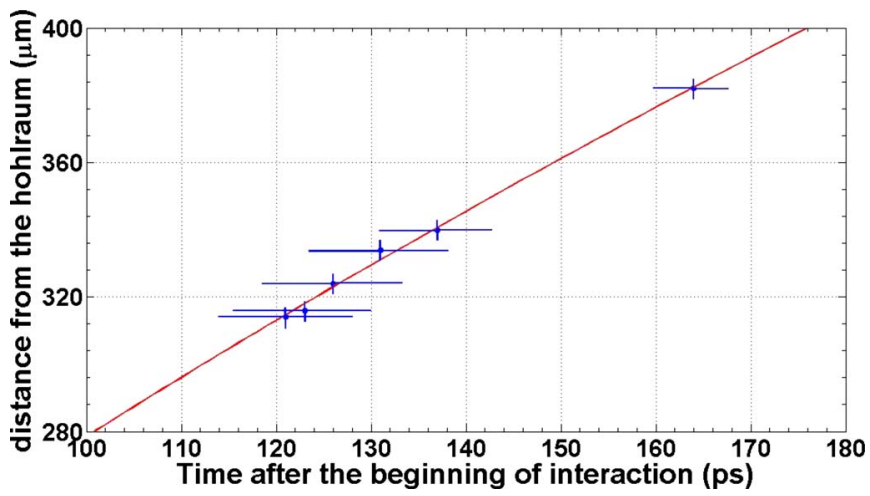

FIG. 3. (Color online) EH propagation as a function of time relative to the beginning of the interaction. The temporal error bars are due to the finite transit time of the protons across the structure. Experimental data indicate a constant velocity of $(1.6 \pm 0.6) \times 10^{6} \mathrm{~m} / \mathrm{s} \simeq(1.6 \pm 0.6) v_{t h}$.

two pronounced depleted regions evidence of the simultaneous presence of two partially overlapping EHs within the structure. The electron depletion is of the order of $\approx 5 \%-$ $10 \%$, well within the range of density depletions seen in published simulations ranging from $1 \%-2 \%$ (Ref. 24) up to $15 \%-20 \% .^{26}$

For EHs in unmagnetized plasmas, the potential profile can be analytically approximated by its small-amplitude expression,

$$
\phi(x)=\phi_{\max } \operatorname{sech} h^{4}\left(\frac{x}{4 \sqrt{\gamma_{e}}}\right),
$$

where $\gamma_{e}$ is a numerical factor ranging from 0 to 1 depending on the electron velocity distribution in the plasma. ${ }^{27} \gamma_{e}=1$ corresponds to a pure Maxwellian distribution while a smaller value of $\gamma_{e}$ implies a larger deviation from the Maxwellian behavior or, equivalently, a smaller value of $\kappa$, following the definition of the $\kappa$-distribution function for nonthermal plasmas. $^{28}$

We stress that the latter analytical expression is valid for weak excitations. Strictly speaking, therefore, it does not apply in our (large-amplitude) case $\left(\phi=\mathrm{eV} / K_{B} T_{e} \simeq 15\right)$ yet is rather to be considered as a first approximation to the experimental results.

The experimental potential shape [Fig. 4(b)] can then be interpreted as the sum of two different bell potentials with $\gamma_{e} \approx 0.7$ corresponding to $\kappa \approx 4$. In the experiment, such a deviation from a Maxwellian distribution may be associated to an incomplete thermalization of the background plasma and/or the effect of superthermal electrons created during the main interaction. The observed structure is then consistent with two partially overlapped EHs with similar amplitude (Fig. 4). It is well known that EHs with similar propagation velocity tend to attract each other and coalesce. ${ }^{15}$ The nonzero electron inertia implies that this process occurs on a time scale of $t_{e} \approx 2 \pi / \omega_{p e} \approx 60 \mathrm{ps}$. The early time at which the structure is detected $\left(\approx 2 t_{e}\right)$ might explain why a full coalescence has not been reached yet.

The perturbation driving the $\mathrm{EH}$ generation is likely to be caused by the sudden charging of the hohlraum walls due to the residual positive charge in the target left behind by
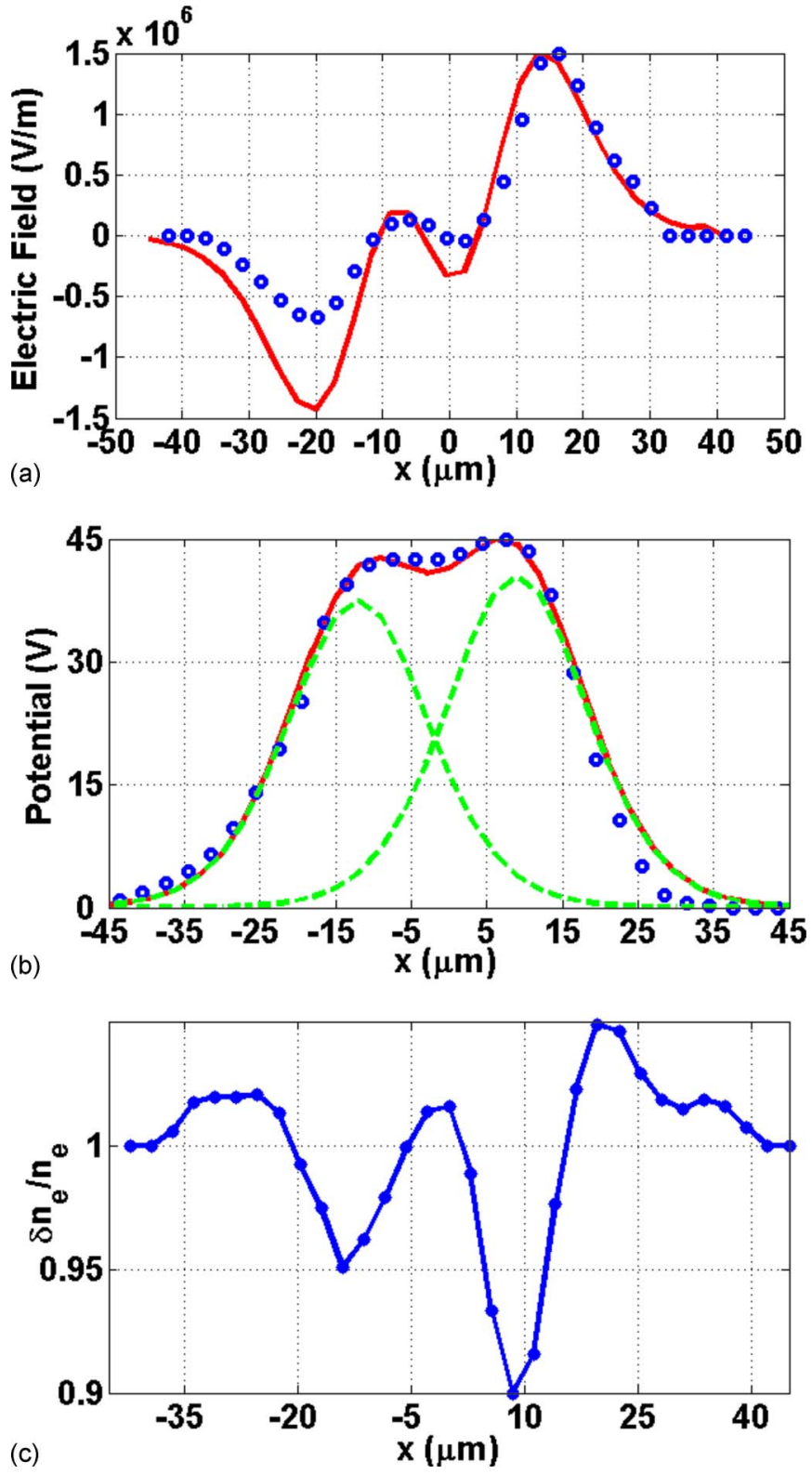

FIG. 4. (Color online) (a) Electric field distribution: experimental electric field distribution across the soliton structure (blue empty circles) compared with the electric field profile (red line) obtained, by using Poisson's equation, from the theoretical potential in (b). (b) Potential spatial profile: experimental potential profile (blue empty circles) compared with the theoretical potential profile (red line) obtained by adding the contribution of two partially overlapping EHs (green dashed curves). (c) Electron density depletion: experimental electron density distribution inside the EHs; two different regions of electron depletion are clearly visible suggesting the presence of two different EH structures.

accelerated electrons that are energetic enough to escape. ${ }^{29}$ This potential can be estimated from the deflection of the probing protons passing close to the walls [highlighted in Fig. 2(a)]. Contiguous regions of proton depletion and accumulation are in fact present in proximity of the walls [Fig. 2(a)] consistent with an electrostatic potential at the wall surface of $\approx 800 \mathrm{~V}=400 K_{B} T_{e} / e$. A significant difference in amplitude between the $\mathrm{EH}$ and the driving potential is reported both in experiments ${ }^{16}$ and simulations ${ }^{24}$ and it thus appears to be a necessary condition for the excitation of such 
structures. It is worth noting that, since the EH is triggered only by the electrostatic potential at the target surface, one can deduce that the geometry and nature of the target itself do not play an essential role.

The experimental propagation velocity of $(1.6 \pm 0.6) v_{t h}$ slightly exceeds the allowed velocity range $v_{\mathrm{EH}} \leq 1.307 v_{t h}$ set by the analytical theory developed by Schamel et al. ${ }^{1}$ However, it has to be noted that this range is valid only for a pure Maxwellian distribution and for EHs that have reached stationarity. The significant deviation from a Maxwellian distribution that the data suggest, together with the very early times at which the structure is observed, could be a possible explanation for this partial disagreement. Indeed, simulations by Califano et al. $^{24}$ show propagation velocities ranging from a fraction of $v_{t h}$ up to $2 v_{t h}$. The extremely high ion Mach number of the structure $(M=530)$, together with the very early times at which the structure is observed, definitely excludes the possibility of an ion wave driven phenomenon such as an ion-acoustic shock. ${ }^{30}$

Published analytical models ${ }^{1}$ and numerical simulations ${ }^{24,26}$ set the conditions to identify a solitary structure in a plasma as an EH to be a positive humplike potential, a depletion in the electron density at the potential peak, and finally a propagation velocity of the order of the thermal electron velocity. The results shown in Figs. 3 and 4 satisfy all the requirements expressed above. Even the spatial width of $\approx 10-12 \lambda_{D}$ concurs with the hypothesis of an EH detection. ${ }^{7,24}$ We have verified that, varying the plasma parameters provided by the simulations by up to an order of magnitude does not invalidate the arguments supporting the EH interpretation, confirming its robustness.

In summary, we have reported the first laboratory observation of laser-created EHs. Thanks to a high temporal and spatial resolution, of the order of few picoseconds and few micrometers, respectively, electric field, potential profile, and electron density distribution have been simultaneously measured in good agreement with published analytical theory and simulations. Data analysis indicates the generation of two partially overlapping EHs moving with a velocity close to the thermal electron velocity; the influence of the nonthermality of the background plasma on the EH features has also been highlighted. The experimental setup shown permits to create and characterize EHs in detail, in either unmagnetized or externally magnetized plasmas. By varying the laser and ambient plasma parameters, it will be in the future possible, using this probing technique, to study the existence, stability, and dynamics of EHs in a broad range of plasma parameters, advancing significantly the knowledge of these structures that play a key role in many different collisionless plasmas scenarios.

Funding for this research has been provided by the AWE Academic Access Scheme, EPSRC Grant Nos. EP/ E035728/1 and EP/C003586/1, by DFG TR 18, GK 1203, and FOR 1048 VR. The work of I.K. was supported by EPSRC-GB Science and Innovation award in Plasma Physics to the Centre for Plasma Physics (Grant No. EP/D06337X/ 1). The authors acknowledge the support of the HELEN
Facility laser and target preparation personnel and K. Quinn for fruitful discussions.

${ }^{1}$ H. Schamel, Phys. Rep. 140, 161 (1986).

${ }^{2}$ M. E. Dieckmann, P. K. Shukla, and B. Eliasson, New J. Phys. 8, 225 (2006).

${ }^{3}$ J. R. Danielson, F. Anderegg, and C. F. Driscoll, Phys. Rev. Lett. 92, 245003 (2004).

${ }^{4}$ J. F. Drake, M. Swisdak, C. Cattell, M. A. Shay, B. N. Rogers, and A. Zeiler, Science 299, 873 (2003).

${ }^{5}$ K. Koyama, R. Petre, E. V. Gotthelf, U. Hwang, M. Matsuura, M. Ozaki, and S. S. Holt, Nature (London) 378, 255 (1995).

${ }^{6}$ F. S. Mozer, R. Ergun, M. Temerin, C. Cattell, J. Dombeck, and J. Wygant, Phys. Rev. Lett. 79, 1281 (1997).

${ }^{7}$ M. Hoshino, Science 299, 834 (2003).

${ }^{8}$ L. Andersson, R. E. Ergun, J. Tao, A. Roux, O. LeContel, V. Angelopoulos, J. Bonnell, J. P. McFadden, D. E. Larson, S. Eriksson, T. Johansson, C. M. Cully, D. L. Newman, M. V. Goldman, K.-H. Glassmeier, and W. Baumjohann, Phys. Rev. Lett. 102, 225004 (2009).

${ }^{9}$ R. E. Ergun, C. W. Carlson, J. P. McFadden, F. S. Mozer, L. Muschietti, I. Roth, and R. J. Strangeway, Phys. Rev. Lett. 81, 826 (1998).

${ }^{10}$ J. S. Pickett, J. D. Menietti, D. A. Gurnett, B. Tsurutani, P. M. Kintner, E. Klatt, and A. Balogh, Nonlinear Process. Geophys. 10, 3 (2003).

${ }^{11}$ C. Cattell, J. Crumley, J. Dombeck, J. R. Wygant, and F. S. Mozer, Geophys. Res. Lett. 29, 1065, doi:10.1029/2001GL014046 (2002).

${ }^{12}$ S. D. Bale, P. J. Kellogg, D. E. Larsen, R. P. Lin, K. Goetz, and R. P. Lepping, Geophys. Res. Lett. 25, 2929, doi:10.1029/98GL02111 (1998).

${ }^{13}$ A. Mangeney, C. Salem, C. Lacombe, J.-L. Bougeret, C. Perche, R. Manning, P. J. Kellogg, K. Goetz, S. J. Monson, and J.-M. Bosqued, Ann. Geophys. 17, 307 (1999).

${ }^{14}$ M. Blaskiewicz, J. Wei, A. Luque, and H. Schamel, Phys. Rev. ST Accel. Beams 7, 044402 (2004).

${ }^{15}$ K. Saeki, P. Michelsen, H. L. Pecseli, and J. J. Rasmussen, Phys. Rev. Lett. 42, 501 (1979).

${ }^{16}$ P. Guio, S. Børve, L. K. S. Daldorff, J. P. Lynon, P. Michelsen, H. L. Pécseli, J. Rasmussen, K. Saeki, and J. Trulsen, Nonlinear Processes Geophys. 10, 75 (2003).

${ }^{17}$ W. Fox, M. Porkolab, J. Egedal, N. Katz, and A. Le, Phys. Rev. Lett. 101, 255003 (2008).

${ }^{18}$ G. Sarri, M. Borghesi, C. A. Cecchetti, L. Romagnani, R. Jung, O. Willi, D. J. Hoarty, R. M. Stevenson, C. R. D. Brown, S. F. James, P. Hobbs, J. Lockyear, S. V. Bulanov, and F. Pegoraro, Eur. Phys. J. D 55, 299 (2009).

${ }^{19}$ L. Romagnani, J. Fuchs, M. Borghesi, P. Antici, P. Audebert, F. Ceccherini, T. Cowan, T. Grismayer, S. Kar, A. Macchi, P. Mora, G. Pretzler, A. Schiavi, T. Toncian, and O. Willi, Phys. Rev. Lett. 95, 195001 (2005).

${ }^{20}$ M. J. Norman, J. E. Andrew, T. H. Bett, R. K. Clifford, J. E. England, N. W. Hopps, K. W. Parker, K. Porter, and M. Stevenson, Appl. Opt. 41, 3497 (2002)

${ }^{21}$ R. A. Snavely, M. H. Key, S. P. Hatchett, T. E. Cowan, M. Roth, T. W. Phillips, M. A. Stoyer, E. A. Henry, T. C. Sangster, M. S. Singh, S. C. Wilks, A. Mackinnon, A. Offenberger, D. M. Pennington, K. Yasuike, A. B. Langdon, B. F. Lasinski, J. Johnson, M. D. Perry, and E. M. Campbell, Phys. Rev. Lett. 85, 2945 (2000).

${ }^{22}$ J. F. Dempsey, D. A. Low, S. Mutic, J. Markman, A. S. Kirov, G. H. Nussbaum, and J. F. Williamson, Med. Phys. 27, 2462 (2000).

${ }^{23}$ M. Borghesi, A. J. Mackinnon, D. H. Campbell, D. G. Hicks, S. Kar, P. K. Patel, D. Price, L. Romagnani, A. Schiavi, and O. Wili, Phys. Rev. Lett. 92, 055003 (2004).

${ }^{24}$ F. Califano and M. Lontano, Phys. Rev. Lett. 95, 245002 (2005).

${ }^{25}$ J. T. Larsen and S. M. Lane, J. Quant. Spectrosc. Radiat. Transf. 51, 179 (1994).

${ }^{26}$ B. Eliasson and P. K. Shukla, Phys. Rev. Lett. 93, 045001 (2004).

${ }^{27}$ M. V. Goldman, D. L. Newman, and A. Mangeney, Phys. Rev. Lett. 99, 145002 (2007).

${ }^{28}$ R. L. Mace and M. A. Hellberg, Phys. Plasmas 2, 2098 (1995).

${ }^{29}$ K. Quinn, P. A. Wilson, C. A. Cecchetti, B. Ramakrishna, L. Romagnani, G. Sarri, L. Lancia, J. Fuchs, A. Pipahl, T. Toncian, O. Wili, R. J. Clarke, D. Neely, M. Notley, P. Gallegos, D. C. Carroll, M. N. Quinn, X. H. Yuan, P. McKenna, T. V. Liseykina, A. Macchi, and M. Borghesi, Phys. Rev. Lett. 102, 194801 (2009).

${ }^{30}$ L. Romagnani, S. V. Bulanov, M. Borghesi, P. Audebert, J. C. Gauthier, K. Lowenbruck, A. J. Mackinnon, P. Patel, G. Pretzler, T. Toncian, and O. Wili, Phys. Rev. Lett. 101, 025004 (2008). 